\title{
Cross Matching of Music and Image
}

\author{
Xixuan Wu'1,2 Yu Qiao ${ }^{1}$, Xiaogang Wang ${ }^{3}$, and Xiaoou Tang ${ }^{1,2}$ \\ ${ }^{1}$ Shenzhen Institutes of Advanced Technology, Chinese Academy of Sciences, China \\ ${ }^{2}$ Department of Information Engineering, The Chinese University of Hong Kong \\ ${ }^{3}$ Department of Electronic Engineering, The Chinese University of Hong Kong \\ \{wxx010,xtang\}@ie.cuhk.edu.hk,yu.qiao@siat.ac.cn,xgwang@ee.cuhk.edu.hk
}

\begin{abstract}
Human perception of music and image are highly correlated. Both of them can inspire human sensation like emotion and power. This paper investigates how to model the relationship between music and image using 47,888 music-image pairs extracted from music videos. We have two basic observations for this relationship: 1) music space exhibits simpler cluster structure than image space, and 2) the relationship between the two spaces is complex and nonlinear. Based on these observations, we develop Multiple Ranking Canonical Correlation Analysis (MR-CCA) to learn such relationship. MR-CCA clusters the music-image pairs according to their music parts, and then conducts Ranking CCA (R-CCA) for each cluster. Compared with classical CCA, R-CCA takes account of the pairwise ranking information available in our dataset. MR-CCA improves performance and significantly reduce computational cost. Experiment results show that R-CCA outperforms CCA, and MR-CCA has the best performance with a consistency score of $84.52 \%$ with human labeling. The proposed method can be generalized to model cross media relationship and has potential applications in video generation, background music recommendation, and joint retrieval of music and image.
\end{abstract}

\section{Categories and Subject Descriptors}

H.3.1 [Information Storage and Retrieval]: Content Analysis and Indexing

\section{General Terms}

Music,Image,Human Factors

\begin{abstract}
This work is partly supported by National Natural Science Foundation of China (61002042), Shenzhen Basic Research Program for Distinguished Young Scholar (JC201005270350A), 100 Talents Programme of Chinese Academy of Sciences, and Introduced Innovative R\&D Team of Guangdong Province "Robot and Intelligent Information Technology", and Shenzhen Key Lab of Computer Vision and Pattern Recognition.
\end{abstract}

Permission to make digital or hard copies of all or part of this work for personal or classroom use is granted without fee provided that copies are not made or distributed for profit or commercial advantage and that copies bear this notice and the full citation on the first page. To copy otherwise, to republish, to post on servers or to redistribute to lists, requires prior specific permission and/or a fee.

WOODSTOCK' 97 El Paso, Texas USA

Copyright 2012 ACM 978-1-4503-1089-5/12/10 ...\$15.00.

\section{Keywords}

Music-image similarity, Ranking Canonical Correlation Analysis, Combination of Clusters

\section{INTRODUCTION}

Music and image are two popular forms of media, one in audio and the other in vision. It is well known that human perceptions of music and image show strong correlations with each other. Both of them can inspire similar human sensation like emotion, power, and weight. Psychology and cognition studies indicate that brain information processing of visual and audio are related[7]. Osborne [8] proposes that music can stimulate visual imagery. Juslin et al.[5] argue that visual imagery is an important mechanism by which music brings emotion. For example, people may react with both positive emotions when listening to melodic movement as 'upward' or facing a beautiful nature scene. In movie and TV program, music and image (video) often appear in parallel as complement to each other to enhance emotion resonance. Meyer [6] discusses that "it seems probable that ... image processes play a role of great importance in the musical affective experiences of many listeners".

There exist works to model the relationship between text and image [9] or text and music [12]. But few works directly analyze the semantic relationship between image and music. To our best knowledge, our work is the first one to investigate the matching degree between image and music.

The objective of this paper is to preliminarily study this challenging problem. To bridge music and image is hard. Firstly, image and music have different feature representations. Secondly, both image space and music space exhibit complex structure, and the relationship between them is nonlinear. This paper develops Multiple Ranking Canonical Correlation Analysis (MR-CCA) to deal with this problem. MR-CCA clusters music-image pairs according to their music sides, and utilizes Ranking CCA to model the local relationship for each cluster. It is noted that the proposed method is general and can be applied to other problems.

To examine the effectiveness of the proposed method, we collected a set of 47,888 music-image pairs from more than 1,500 music videos. We chose half of these pairs and asked labelers to compare their matching degree. The labelers largely agree with each other on the annotation, which indicates that human have consensus on this problem. The experimental results of our method show that MR-CCA has the best performance and achieves a consistency score of $84.52 \%$ with human annotation. 


\section{PREPARATIONS}

This section describes how to construct and annotate the dataset used in this paper, and then discuss how to calculate feature representation for music and image.

\subsection{Data and annotation}

We collected a set of 47,888 music-image pairs from more than 1,500 music videos (MVs), which cover music with various styles and genres, and are from about 500 singers. Since a MV contains audio signal with large variation, different audio segments can correspond to different images. So we segment the music with dynamic texture model [1], and each music-image pair is composed of a key frame from the video part and its associated music segment. Since most music videos are created by experts with deliberate consideration, most pairs obtained in this way should be well matched. To further validate the dataset, we ask human labelers to compare the matching degree of a pair from MV and a random pair (music is the same but image is selected randomly). Al1 labelers prefer music-image pair from MV with a rate of $89.2 \%$.

\subsection{Representation of music and image}

Two categories of features are considered for music representations. The first is the Echo Nest Song(ENS) feature vector, each dimension of which corresponds to a mid-level acoustic characteristic [11] such as tempo, mode, and mean section length. There exists open API to calculate ENS features $^{1}$. The second category consists of a set of semantic features, each of which corresponds to a semantic word description of a song, like happy or relax. The semantic words are obtained from famous music sites, such as Google music. A music segment is represented as a vector of posterior probabilities with respect to the pre-defined semantic words, computed with mix hierarchical learning [12]. Mainly following [10], image is represented by three types of features, 1) color features: hue and RGB histogram, color SIFT, color spatialet etc; 2) shape features: Histogram of Gradient, edge orientation histogram etc; 3)texture and appearance features: wavelet, GIST etc. The music and image feature vector has a total dimension of 133 and 2,596, respectively. For each type of music and image feature, we define a distance function for it.

\section{MULTIPLE RANKING CANONICAL COR- RELATION ANALYSIS}

In this section, we introduce Multiple Ranking Canonical Correlation Analysis (MR-CCA). MR-CCA is a learning based approach and its diagram is shown in Fig. 1. Let $V_{i}=\left\{I_{i}, M_{i}\right\}$ denote a set of music-image pairs for training. The objective of MR-CCA is to estimate a similarity function $\mathcal{S}(I, M)$ between image $I$ and music $M$.

\subsection{Cluster and Distance to Reference Trans- formation}

Both music and image space have high dimension and complex structures. To simplify the problem, we cluster music-image pairs first. It is believed that the music-image relationship in each cluster should be simpler and more easily to be modeled. One problem is that the relationship

\footnotetext{
${ }^{1}$ http://developer.echonest.com
}

between music and image is many-to-many not one-to-one. So it is not a good idea to cluster pairs with both music and image features. Compared with image space, music space includes less diversity and exhibits simpler cluster structure. Thus, we use normalized cut to cluster the music parts, and the images are divided according to the cluster information of their music parts. Let $\left\{V_{1}, V_{2}, \ldots, V_{C}\right\}$ denote the clusters.

Inspired by the success of similarity based representation in $[2,13]$, we use a Distance to Reference Transformation (DtRT) which converts original feature into a new DtRT representation. For each cluster $V_{c}$, we select a set of reference samples $\left\{I_{c}^{r}, M_{c}^{r}\right\}_{r=1}^{R}$ where $R$ is the size of reference set. For another music $I$ in $V_{c}$, we calculate distance $d_{I}\left(I, I_{c}^{r}\right)$, then convert the distance to similarity by $s_{c}^{r}(I)=$ $\exp \left\{-\frac{d_{I}\left(I, I_{c}^{r}\right)^{2}}{\sigma_{I}{ }^{2}}\right\}$, where $\sigma_{I}$ is a normalization parameter. The new representation for image $I$ is defined as a vector composed by the similarities $x_{c}(I)=\left[s_{c}^{1}(I), \ldots, s_{c}^{R}(I)\right]$. In the same way, we can get $y_{c}(M)=\left[s_{c}^{1}(M), \ldots, s_{c}^{R}(M)\right]$ for music $M$. In the next, we use DtRT representation $x_{c}(I), y_{c}(M)$ (or $x, y$ for simplicity) instead of $I, M$, and set similarity function $\mathcal{S}(x, y)=\mathcal{S}(I, M)$. One advantage of using $x$ and $y$ is that both of them have the same dimension, and their components are aligned correspondingly.

\subsection{Ranking Canonical Correlation Analysis}

This section proposes Ranking CCA (R-CCA) to estimate a similarity function $S_{c}(x, y)$ for each cluster $c$. Let $V_{c}=\left\{x_{i}, y_{i}\right\}$ denote the set of training pairs in cluster $c$. Canonical correlation analysis (CCA) [4] aims to find data projections with the largest correlation across two (or more) spaces, which has been successfully used for crossmodal multimedia retrieval [9]. Introduce the project matrices $A=\left[a_{1} ; a_{2} ; \ldots ; a_{J}\right], B=\left[b_{1} ; b_{2} ; \ldots ; b_{J}\right]$. The objective of CCA is $\max _{A, B} \sum_{j=1}^{J} \sum_{i=1}^{N} a_{j} x_{i} b_{j} y_{i}$. It can be shown that optimal $A$ are composed by the eigenvectors of $\Sigma_{X X}^{-1} \Sigma_{X Y}$ $\Sigma_{Y Y}^{-1} \Sigma_{Y X}$ associated with the $J$ largest eigenvalues, while $B$ are composed by the eigenvectors of $\Sigma_{Y Y}^{-1} \Sigma_{Y X} \Sigma_{X X}^{-1} \Sigma_{X Y}$ associated with the $J$ largest eigenvalues, where $\Sigma_{X X}, \Sigma_{Y Y}$ are covariance matrices for $x_{i}, y_{i}$ respectively, and $\Sigma_{X Y}, \Sigma_{Y X}$ are covariance matrices between $x_{i}$ and $y_{i}$. With $A, B$, the similarity function of CCA is defined by,

$$
\mathcal{S}_{c}^{\mathrm{CCA}}(x, y)=\langle A x, B y\rangle .
$$

Human annotation described in Section 2.1 yields pairwise ranking information, which means one pair should have higher similarity score than another, i.e., $\mathcal{S}\left(x_{i}, y_{i}\right)>\mathcal{S}\left(x_{i}^{\prime}, y_{i}^{\prime}\right)$. Classical CCA cannot handle such kind of pairwise ranking information. Here we develop Ranking Canonical Correlation Analysis (R-CCA) to take account of these information. Mathematically, the objective of R-CCA learning is,

$$
\min _{A, B} \sum_{i} f\left(\mathcal{S}_{c}\left(x_{i}, y_{i}\right)-\mathcal{S}_{c}\left(x_{i}^{\prime}, y_{i}^{\prime}\right)\right)
$$

where similarity function $\mathcal{S}_{c}\left(x_{i}, y_{i}\right)$, has the same form as Eq. 1 , and $f$ is a hinge penalty function. If $t \leq 0, f(t)=-t$; otherwise $f(t)=0$. The constraints are $\left\|a_{i} X\right\|=\left\|b_{i} Y\right\|=1$, $a_{i}^{T} X^{T} X a_{j}=0$, and $b_{i}^{T} Y^{T} Y b_{j}=0$.

To directly optimize Eq. 2 is difficult. This is because $\mathcal{S}$ has a quadratic form of $A$ and $B$, and both $A$ and $B$ contain a large number of variables. To simplify the problem, we apply classical CCA on matched pairs $\left\{\left(x_{i}, y_{i}\right)\right\}$ to obtain projection matrices $A$ and $B$ to reduce the dimensionality, 


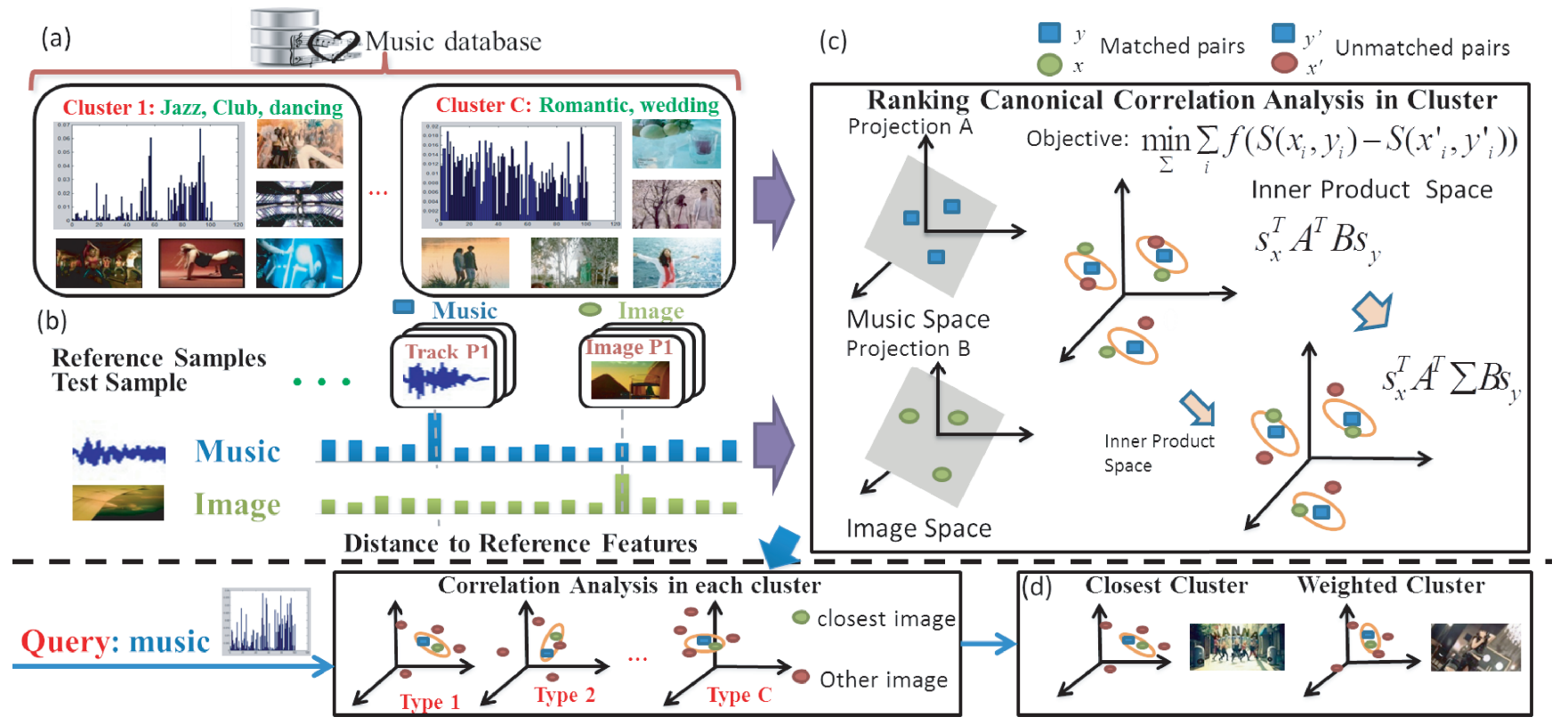

Figure 1: Framework of Multiple Ranking Canonical Correlation Analysis: (a). Cluster in music space; (b). DtRT representation for music and image; (c). Ranking canonical correlation analysis in cluster; (d). Combination of estimation score of clusters.

and introduce the following similarity function for R-CCA,

$$
\mathcal{S}_{c}^{\mathrm{R}-\mathrm{CCA}}\left(x_{i}, y_{i}\right)=x_{i}^{\top} A^{\top} \Sigma B y_{i},
$$

where $\Sigma$ is a matrix with size $J \times J$. Since $A$ and $B$ are known, we only need to optimize $\Sigma$ which minimizes Eq. 2.

$\Sigma$ contains much less variables than $A, B$. Moreover, Eq. 3 has a linear form of $\Sigma$ which makes optimization easier. We can further assume $\Sigma$ is a diagonal matrix, since the projections obtained by CCA are uncorrelated. Let $W=$ $\left[w_{1}, w_{2}, \ldots, w_{J}\right]$ denote the diagonal of $\Sigma$. Introduce variables, $z_{i}^{j}=a_{j} x_{i} b_{j} y_{i}, z_{i}=\left[z_{i}^{1}, z_{i}^{2}, \ldots, z_{i}^{J}\right], z_{i}^{j^{\prime}}=a_{j} x_{i}{ }^{\prime} b_{j} y_{i}{ }^{\prime}$, and $z_{i}^{\prime}=\left[z_{i}^{1^{\prime}}, z_{i}^{2^{\prime}}, \ldots, z_{i}^{J^{\prime}}\right]$. Then Eq. 3 can be written into,

$$
\mathcal{S}_{c}^{\mathrm{R}-\mathrm{CCA}}\left(x_{i}, y_{i}\right)=\sum_{j} w_{j} a_{j} x_{i} b_{j} y_{i}=W^{\top} z_{i} .
$$

Then our objective reduces to optimize $W$ with

$$
\min _{W} \sum_{i} f\left(W^{\top} z_{i}-W^{\top} z_{i}^{\prime}\right) .
$$

This is in spirit the same as the optimization for ordinal SVM [3], whose due problem is defined as

$$
\min _{W}\|W\|^{2}+\sum \xi_{i}
$$

subject to,

$$
\begin{aligned}
\xi_{i} & \geq 0, \\
W^{T} z_{i}-W^{T} z_{i}^{\prime} & \geq 1-\xi_{i} .
\end{aligned}
$$

The above problem is a quadratic programming which can be solved by Lagrangian multipliers.

\subsection{Similarity score ensemble}

In previous subsection, we have estimated a similarity function for each cluster by CCA (Eq. 1) or R-CCA (Eq. 3). For a testing pair $(x, y)$, we need to combine these similarity functions to obtain a final similarity score. The simple idea is to determine which cluster $(x, y)$ belongs to. Let $c *$ denote the index of the nearest cluster. Then

$$
\mathcal{S}(x, y)=\mathcal{S}_{c *}(x, y) .
$$

Eq. 9 is simple. But it cannot deal well with the samples near cluster boundary. To overcome this problem, we introduce a 'soft' similarity function by using softmax function as weights. Let $d_{c}$ denote the distance between pair $(x, y)$ and the center of $c$-th cluster. The weighted similarity function is,

$$
\mathcal{S}(x, y)=\sum_{c=1}^{C} \frac{\exp \left(-d_{c} / \sigma^{2}\right)}{\sum_{j=1}^{C} \exp \left(-d_{j} / \sigma^{2}\right)} \mathcal{S}_{c}(x, y),
$$

where $C$ is the number of cluster, and $\sigma$ is a normalization parameter. Eq. 10 can model the nonlinear relation between $x$ and $y$, since weights depend on input pair $(x, y)$.

\section{EXPERIMENT}

This section presents our experimental evaluation. We randomly select 20,000 of all 22,632 music segments for clustering and training, and use the rest for testing. We randomly select a set of samples from each cluster as its reference set. We explore the effect of two parameters, the number of cluster $K$ and the proportion of reference set size to cluster size $1 / d$. $K$ is changed from 3 to 20 . $d$ is changed from 2 to 20 , which means $1 / d$-th samples in each cluster are used as the corresponding reference set. The labelers' preference are set as ground truth. The consistency rates (precision) with human labeling are used as evaluation criterion. We repeat each experiment 10 times.

Comparison of Methods. We compared three similarity estimation methods, closest CCA (C-CCA, Eq. 1+Eq. 9), closest Ranking CCA (CR-CCA, Eq. 4+Eq. 9), and Multiple Ranking CCA (MR-CCA, Eq. 4+Eq. 10). Since there is no previous study on this problem, closest CCA can be seen as a baseline method. Firstly, we fix the proportion of reference set $1 / d$ as $1 / 6$ and, change the number of 


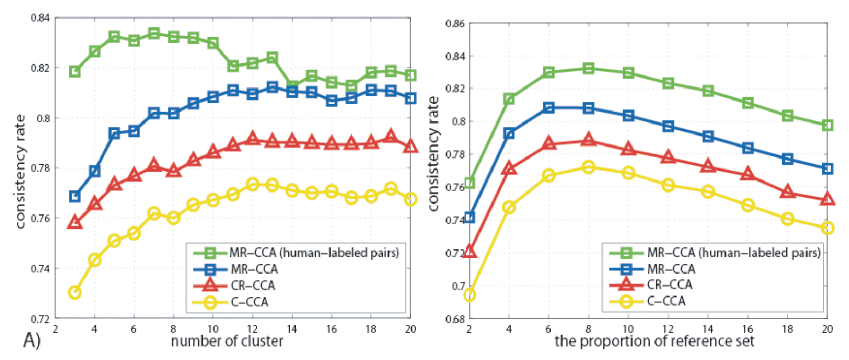

Figure 2: A) Precisions using C-CCA, CR-CCA, MR-CCA and MR-CCA with human-labeled pairs when changing the number of cluster; B) Precisions using C-CCA, CR-CCA, MR-CCA and MR-CCA with human-labeled pairs when changing the proportion of reference set to training set, $x$ axis is the inverse of the proportion.

Table 1: Local reference Vs Global reference

\begin{tabular}{|c|c|l|}
\hline Reference Type & Consistency Rate & Training Time Cost \\
\hline Local reference & $80.35 \%$ & $46.3999 \mathrm{~s}$ \\
\hline Global reference & $79.24 \%$ & $869.2618 \mathrm{~s}$ \\
\hline
\end{tabular}

cluster $K$. Experimental results are shown in Fig. 2A. The precisions of all three methods generally increase with the number of cluster when $K \leq 10$. This is partly because too few clusters cannot model the whole space precisely. When $K>10$, the precisions increase little. The highest precision we obtained is $81.23 \%$, when the number of cluster is 13 and MR-CCA is used. We also conduct an experiment that only uses some human-labeled pairs to test. It shows that our methods perform better on average. The highest precision is $84.52 \%$ with MR-CCA when $K=5$ and $d=5$. This means our methods are comparable with human annotation performance on this task.

Secondly, we fix the number of cluster $K$ as 10 and change the proportion of reference set $1 / d$. The results are depicted in Fig. 2B. The best $1 / d$ is around $1 / 6 \sim 1 / 8$. This is because DtRT features cannot convey enough information when too few references are used, while too many reference can lead to redundant and noisy representations.

In all our experiments, we find MR-CCA archives the best performance among the three methods, and CR-CCA always outperforms C-CCA. This indicates that 1)Ranking CCA is more effective than classical CCA due to the fact that it can use pairwise ranking information in training, and 2) the weighted summation of similarity functions (Eq. 10) is better than the single closest similarity function (Eq. 9).

Comparison of Time Efficiency. Another issue is how to select reference samples. In spite of selecting local references for each cluster, one may suggest to use global reference set which is randomly chosen from the whole training set. In the next, we make comparison between local reference and global reference. To be fair for both kinds of reference, we fixe the proportion of reference set as $1 / 10$. Cluster number $K$ is chosen as 10 . We also conduct experiments with other $d$ and $K$. The tendency is similar, thus we omit these results due to space limitation. The precisions and training time cost are shown in Table 1 . All the experiments are run on a machine with $2.80 \mathrm{GHz} \mathrm{CPU}$ and 16GB of RAM. It can be seen that local reference has slightly better performance and is much faster, which is more scalable for larger or distributed database. That's because the DtRT feature representations $x, y$ has lower dimension with local reference than with global reference for the same $d$.

\section{CONCLUSIONS}

This paper develops multiple ranking CCA which considers both cluster structure and pairwise ranking information to learn similarity between music and image. Our experiments on 47,888 pairs show the effectiveness of the proposed methods, and MR-CCA achieves a consistency score of $84.52 \%$ with human labelers on comparing music-image pairs. It is noted that to model the relationship between music and image is a challenging and general problem. This paper is more of an attempt to introduce the topic and present methods with inspiring results on data from music video. Although the discussions are limited to music-image pairs, our analysis and methods can be generalized to other type$\mathrm{s}$ of multimodal relationship. The proposed methods have been successfully applied to automatic music video generation [14].

\section{REFERENCES}

[1] L. Barrington, A. Chan, and G. Lanckriet. Modeling music as a dynamic texture. IEEE Trans. on ASLP, 2010.

[2] E. Bart and S. Ullman. Single-example learning of novel classes using representation by similarity. In BMVC, 2005.

[3] R. Herbrich, T. Graepel, and K. Obermayer. Support vector learning for ordinal regression. In ICANN, 1999.

[4] R. Johnson and D. Wichern. Applied multivariate statistical analysis. 1992.

[5] P. Juslin and D. Västfjäll. Emotional responses to music: The need to consider underlying mechanisms. Behavioral and Brain Sciences, 2008.

[6] L. Meyer. Emotion and meaning in music. University of Chicago Press, 1961.

[7] I. Olson, J. Gatenby, and J. Gore. A comparison of bound and unbound audio-visual information processing in the human cerebral cortex. Cognitive Brain Research, 2002.

[8] J. Osborne. The mapping of thoughts, emotions, sensations, and images as responses to music. Journal of Mental Imagery, 1981.

[9] N. Rasiwasia, J. Costa Pereira, E. Coviello, G. Doyle, G. Lanckriet, R. Levy, and N. Vasconcelos. A new approach to cross-modal multimedia retrieval. In ACM-MM, 2010.

[10] X. Tang, K. Liu, J. Cui, F. Wen, and X. Wang. Intentsearch: Capturing user intention for one-click internet image search. IEEE Trans. on PAMI, 2011.

[11] D. Tingle, Y. Kim, and D. Turnbull. Exploring automatic music annotation with "acoustically-objective" tags. In ACM-MIR, 2010.

[12] D. Turnbull, L. Barrington, D. Torres, and G. Lanckriet. Semantic annotation and retrieval of music and sound effects. IEEE Trans. on ASLP, 2008.

[13] X. Wang, K. Liu, and X. Tang. Query-specific visual semantic spaces for web image re-ranking. In $C V P R$, 2011.

[14] X. Wu, B. Xu, Y. Qiao, and X. Tang. Automatic music video generation: Cross matching of music and image. ACMMM, 2012. 